\title{
Surgical Treatment Approaches in Severe Spinal Deformities Associated with Intraspinal Pathologies
}

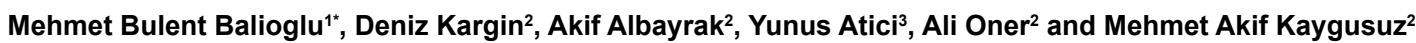

${ }^{1}$ Department of Orthopaedic Surgery, Istinye University Liv Hospital, Istanbul, Turkey

${ }^{2}$ Department of Orthopaedic Surgery, Health Science University Baltalimani Bone, Diseases Education and Research Hospital, Istanbul, Turkey

${ }^{3}$ Faculty of medicine, Department of Orthopaedic Surgery, Okan University, Istanbul, Turkey

\begin{abstract}
Objective: Scoliosis with associated intraspinal anomalies may be treated either before the correction of spinal deformities or during the same session. Our study elucidates the impact of the timing of single- or two-stage neurosurgical and deformity treatment of intraspinal pathologies with the outcomes of serious spinal deformities and discusses the preferable method.
\end{abstract}

Methods: Patients who were operated either concurrently or in two stages, due to intraspinal anomalies associated with rigid spinal deformities, were radiologically and clinically examined. Patients' ages during the neurosurgical treatment and at the time of deformity treatment, period between two surgeries, follow-up period, clinical and radiological results and encountered complications were recorded.

Results: Nineteen patients ( 13 females, 6 males) underwent surgery for spinal deformities associated with intraspinal pathologies between 2007 and 2014. Fifteen (78.9\%) patients underwent a two-stage surgery and four (21.1\%) patients' concurrent surgeries. Mean age of the patients at the time of intraspinal pathology surgery was $8.6 \pm 6.9$ years and at posterior spinal fusion (PSF) $13.4 \pm 3.9$ years. The period between the two surgeries was $54.2 \pm 67.5$ months on average and the mean follow-up period was $39.8 \pm 22.2$ months. The anteroposterior Cobb's angle was measured as $68.2^{\circ} \pm 27.1^{\circ}$ preoperatively and $29.1^{\circ} \pm 18.7^{\circ}$ at final examination $(\mathrm{p}=0.00)$. Visual analog scale score was $8.1 \pm 1$ preoperatively and $1.1 \pm 0.2$ at the final follow-up $(\mathrm{p}=0.00)$.

Conclusion: The etiology, extent of deformity, curve progression and patient's age were indicative in the surgical treatment of intraspinal pathologies and spinal curves. Concurrent surgical interventions may be recommended to avoid additional complications and for quicker recovery.

Keywords: Concurrent surgery; Congenial scoliosis; Congenital spinal pathologies; Kyphosis; Posterior spinal fusion

\section{Abbreviations}

PSF: posterior spinal fusion; CM: Chiari malformation; CM-1: Chiari malformation Type 1; SCM: split cord malformation; NIOM: neurophysiologic intraoperative monitoring; MRI: magnetic resonance imaging; VAS: visual analog scale; TK: thoracic kyphosis; LL: Lumbar lordosis; AP: anteroposterior; PFD: posterior fossa decompression; VCR: vertebral column resection; PSO: pedicle subtraction osteotomies; CFL: cerebrospinal fluid leakage; AIS: adolescent idiopathic scoliosis; PO: Ponte Osteotomy; GFSI: Growth Friendly Spinal Instrumentation.

\section{Introduction}

The prevalence of intraspinal anomalies together with congenital, early onset scoliosis, neuromuscular causes, neurofibromatosis, and connective tissue diseases varies according to etiologies and has been reported as high as 35 to $40 \%$ [1-3]. It is known that the prevalence of intraspinal anomalies increases together with abnormal neurological findings, left side curvature and apical kyphosis [4,5]. The most common intraspinal anomalies observed with scoliosis are Chiari malformation (CM) and syringomyelia [4]. It has been shown that CM patients exhibit an incidence of 50 to $90 \%$ and Chiari malformation Type I (CM-I) patients 15 to $80 \%$ scoliosis [5-9]. Tethered cord associated with lipoma, split cord malformation (SCM) or fatty filum terminale are the other most common intraspinal anomalies. If intraspinal pathology is present during the correction of scoliosis, then the risk of neurological injury may increase together with cord tethering [9]. A complete neurological-neurosurgical assessment is required before the treatment of spinal deformities; if an intraspinal anomaly is present, then the neural axis malformations must be treated first due to the risk of neurological deformation [10,11]. Distal cord tethering may occur together with idiopathic anomalies, lipoma type of intraspinal anomalies or due to cohesion during the repair of dysraphism in myelodysplasia. Neurophysiologic intraoperative monitoring (NIOM) is beneficial for the assessment of cord function during the correction of spinal deformities to prevent idiopathic injury of the nerve root or the spinal cord during release procedures [12]. If scoliosis is associated with tethered cord, then it is recommended to release the affected cord, particularly in younger patients, in order to stop or correct the scoliosis [13]. The possibility of tethered cord occurring together with CM-I has been reported as $14 \%[14,15]$. Therefore, it has been recommended that intraspinal anomalies should be addressed before surgical decompression of CM [14-17].

Our study elucidates the impact of the timing of single- or twostage neurosurgical and deformity treatment of intraspinal pathologies with the outcomes of serious spinal deformities and discusses the preferable method.

*Corresponding author: Mehmet Bulent Balioglu, Department of Orthopaedic Surgery, Istinye University Liv Hospital, Istanbul, Tel: + 905322521483; E-mail: mbbalibey@gmail.com

Received June 01, 2018; Accepted June 12, 2018; Published June 15, 2018

Citation: Balioglu MB, Kargin D, Albayrak A, Atici Y, Oner A, et al. (2018) Surgical Treatment Approaches in Severe Spinal Deformities Associated with Intraspinal Pathologies. J Spine 7: 417. doi: 10.4172/2165-7939.1000417

Copyright: (c) 2018 Balioglu MB, et al. This is an open-access article distributed under the terms of the Creative Commons Attribution License, which permits unrestricted use, distribution, and reproduction in any medium, provided the original author and source are credited. 


\section{Materials and Methods}

Patients who were operated concurrently or in two stages (due to intraspinal anomalies associated with rigid spinal deformities) were retrospectively examined. Intraspinal anomalies were identified using magnetic resonance imaging (MRI). Spinal deformities were examined in both planes by measuring the Cobb angle, together with the associated radiological and clinical pathologies. Patients with spinal deformities and intraspinal pathologies were treated with either neurosurgical intervention at first, followed by a deformity correction at another session, or they were performed concurrently. Posterior long instrumentation and fusion were performed following the treatment of intraspinal anomalies or during the same session. Age of the patients at neurosurgery, deformity correction and fusion age, the period between two surgeries, follow-up period, degree of deformity before the treatment and at the final follow-up, amount of correction, visual analog scale (VAS) score, onset of clinical and neurological findings, clinical and radiological results at the final follow-up and complications were recorded (Table 1).

Descriptive statistics included the mean, standard deviation, median, minimum, maximum, frequency values and ratios. Distribution of the variables was evaluated using the Kolmogorov-Smirnov test. The Wilcoxon and paired sample t-tests were used in analyzing the repeated measurements. SPSS Statistics for Windows v.22.0 (IBM Corp., New York, NY, USA) software was used for statistical analyses.

\section{Results}

Nineteen patients ( 13 females, 6 males) who successively underwent surgery for spinal deformities associated with intraspinal pathologies between 2007 and 2014 were retrospectively evaluated. Mean age of the patients at the intraspinal pathology surgery was $8.6 \pm 6.9$ (median: 12, range: 0.1 to 18.5 ) years and at the posterior instrumentation and correction surgery $13.4 \pm 3.9$ (median: 13.5 , range: 2.8 to 19 ) years. The period between the two surgeries was $54.2 \pm 67.5$ (median: 24 , range: 0 to 228 ) months on average and the mean follow-up period was 39.8 \pm 22.2 (median 31, range: 15 to 90 ) months. On the anteroposterior (AP) plane, the preoperative Cobb angle with a mean of $68.2^{\circ} \pm 27.1^{\circ}$ (median: $70.0^{\circ}$, range: $22^{\circ}$ to $109^{\circ}$ ) was measured significantly lower at the final follow-up as $29.1^{\circ} \pm 18.7^{\circ}$ (median: $24.0^{\circ}$, range: $5^{\circ}$ to $72^{\circ}$; $\mathrm{p}=0.00$ ). On the sagittal plane, the thoracic kyphosis (TK) Cobb angle was preoperatively measured $40.0^{\circ} \pm 36.5^{\circ}$ (median: $49.0^{\circ}$, range: $-34^{\circ}$ to $100^{\circ}$ ) on average and there was no significant difference with the final follow-up values of $35.8^{\circ} \pm 18.3^{\circ}$ (median: $33.0^{\circ}$, range: $-10.0^{\circ}$ to $63.0^{\circ}$; $\mathrm{p}=0.51)$. Lumbar lordosis (LL) was preoperatively measured $-36.9^{\circ} \pm$ 57 (median: $-54.0^{\circ}$, range: $-94.0^{\circ}$ to $86.0^{\circ}$ ) and showed no significant change at final examination with a mean of $-37.7^{\circ} \pm 16.0$ (median: -41.0 , range: $-65.0^{\circ}$ to $-2.0^{\circ} ; \mathrm{p}=0.39$ ). The VAS score was $8.1 \pm 1$ preoperatively and $1.1 \pm 0.2$ at the final follow-up $(\mathrm{p}=0.00)$. Fifteen of the patients (78.9\%) had a two-stage surgery while four $(21.1 \%)$ had concurrent operations for both intraspinal anomalies and spinal deformities. There was no significant difference between the VAS scores of both groups at the final follow-up ( $>>0.05)$. MRI findings of intraspinal anomalies treated with surgery are tethered cord release, myelomeningocele closure; posterior fossa decompression (PFD), intraspinal tumor/cyst excision, and intraspinal bone spur excision. Complications of the surgeries include intraoperative dural damage, pressure soreness on chin, perioperative cardiac arrest, temporary neuromonitorization loss, postoperative implant failure, and wound site problems.

Regarding the two patients with scoliosis and kyphosis associated with CM, a two-stage surgery was performed in one case (the period between PFD and PSF was 2 years) and concurrent surgery was performed on the other. Mean age of the patients during neurosurgery treatment of the two male patients, who were treated with PFD for CMI, was 16.2 (range: 15.9 to 16.6 ) years and at the posterior fusion and deformity correction 17.2 (range: 16.6 to 17.9 ) years. Mean follow-up period was noted as 4.4 (range: 4 to 4.7 ) years. The mean AP Cobb angle was preoperatively measured $66.2^{\circ}$ (range: $40^{\circ}$ to $100^{\circ}$ ) while it was $19.7^{\circ}$ (range: $10^{\circ}$ to $32^{\circ}$ ) at final examination with $70.4 \%$ (range: $61.5 \%$ to $75 \%$ ) correction. On the sagittal plane, the preoperative TK was $64.5^{\circ}$ (range: $62^{\circ}$ to $67^{\circ}$ ) on average, which decreased to $38.5^{\circ}$ (range: $33^{\circ}$ to $44^{\circ}$ ) during the final follow-up with $39.9 \%$ (range: $29 \%$ to $50.8 \%$ ) correction. None of the cases developed any postoperative complications. Of the fifteen patients suffering from spinal deformity associated with tethered cord; ten were treated with tethered cord release (eight in two stages, two with concurrent procedures); the procedure was performed twice on four patients. The correction of spinal deformity was concurrently performed on a patient with congenital scoliosis and on a patient with myelomeningocele. Of the ten patients with tethered cord release (eight females, two males), the mean age was 9.3 (range: 0.3 to 18.5 ) years during neurosurgery (first surgery) and 13.1 (range: 2.8 to 18.5 ) years at posterior fusion and deformity correction. The period between the two surgeries was 45.9 (range: 0 to 156) months on average and the mean follow-up period was recorded as 3 (range: 1.2 to 6.3) years. On MR images, four patients had tethered cords alone whereas six cases had additional intraspinal anomalies; SCM (Type I in four patients and Type II in two patients) was associated with syringomyelia (three patients) and myelodysplasia. In six cases, osteotomy from the posterior to the vertebra (one vertebral column resection [VCR], three pedicle subtraction osteotomies [PSO], and two Ponte osteotomies) was performed for the correction of rigid congenital spinal deformity (Figure 1). The preoperative AP Cobb angle of $62.6^{\circ}$ (range: $25^{\circ}$ to $96.4^{\circ}$ ) decreased to $24.8^{\circ}$ (range: $5^{\circ}$ to $66^{\circ}$ ) at the final follow-up with $60.4 \%$ correction. On the sagittal plane, the TK angle was preoperatively $42.4^{\circ}$ (range: $-6^{\circ}$ to $100^{\circ}$ ) and $37.2^{\circ}$ (range: $-9^{\circ}$ to $60^{\circ}$ ) at the final visit. Mean LL was measured $-39.0^{\circ}$ (range: $86^{\circ}$ to $-94^{\circ}$ ) preoperatively and $-36.6^{\circ}$ (range: $-2^{\circ}$ to $65^{\circ}$ ) at the final follow-up.

The sacs in six of the seven patients suffering from myelodysplasia (spina bifida, myelomeningocele; four females and two males) were closed after birth, shunts were placed in three and tethered cords were released in three of them. The patients were treated with neurosurgery for intraspinal pathologies first, and then with posterior instrumentation and correction at another session for spinal deformity. In one case, release was done twice for the associated tethered cord; the first release was performed while the myelomeningocele was being closed and the second one together with the spinal deformity correction. In one case, both surgical interventions took place during the same session. In the three myelodysplasia patients who had a high risk of problems with the surgical wound site, soft tissue/skin relocation was performed together with plastic and reconstructive surgery during the last operation. Mean age of the patients was 2.1 (range: 0.1 to 12.3 ) months during the neurosurgery intervention and 13.7 (range: 10.7 to 19 ) years at posterior instrumentation and deformity correction. The period between the two surgeries was 124.7 (range: 1 to 228) months and the mean duration of follow-up was 2.7 (range: 1.2 to 7.5 ) years. Three patients had scoliosis, two had kyphosis and two had serious kyphoscoliosis deformities. Posterior vertebra osteotomies were performed in four cases (one VCR and three PSOs) (Figure 1). The preoperative AP Cobb angle of $69.5^{\circ}$ (range: $22^{\circ}$ to $109^{\circ}$ ) decreased to $39.6^{\circ}$ (range: $11^{\circ}$ to $72^{\circ}$ ) at the final follow-up with $43 \%$ correction. On the sagittal plane, the TK was $8.6^{\circ}$ (range: $-34^{\circ}$ to $83^{\circ}$ ) and LL $17.8^{\circ}$ (range: $-80^{\circ}$ to $86^{\circ}$ ) preoperatively. The same parameters were measured $22.4^{\circ}$ (range: $-10^{\circ}$ to $51^{\circ}$ ) and $-27.4^{\circ}$ (range: $-46^{\circ}$ to $-2^{\circ}$ ) during the final visit, respectively. Dural 


\begin{tabular}{|c|c|c|c|c|c|c|c|c|c|}
\hline \multirow{3}{*}{ 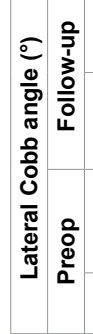 } & 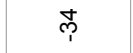 & ț & q & $\hat{\varphi}$ & $\stackrel{6}{\longrightarrow}$ & $\stackrel{\Upsilon}{\varphi}$ & $\stackrel{\oplus}{?}$ & f & $\stackrel{\varphi}{~}$ \\
\hline & $\sigma$ & $\stackrel{\infty}{q}$ & 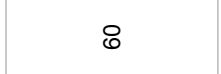 & $m$ & $\tilde{ల}$ & $\ddot{\circ}$ & 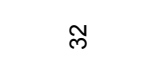 & $\grave{N}$ & $\stackrel{\infty}{\infty}$ \\
\hline & ใุ & $\hat{T}$ & \& & ه & $\stackrel{\infty}{+}$ & ?ִ & ț & ?. & 节 \\
\hline$\breve{F}$ & $\bar{\sigma}$ & ৪ & ๗ & $\wedge$ & i & $\stackrel{\infty}{+}$ & $\bar{m}$ & $F$ & 움 \\
\hline 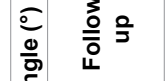 & $\stackrel{\infty}{\sim}$ & $\stackrel{m}{=}$ & 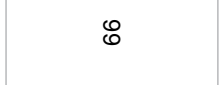 & is & ম & A & $\bar{N}$ & 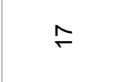 & A \\
\hline 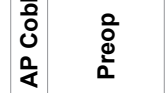 & \&े & m & $\mathscr{8}$ & 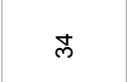 & $\mathscr{\circ}$ & $\bar{\sigma}$ & N & 8 & $\stackrel{8}{2}$ \\
\hline 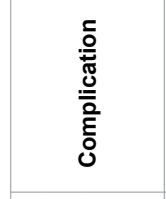 & 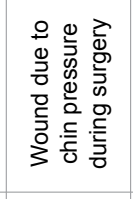 & : & 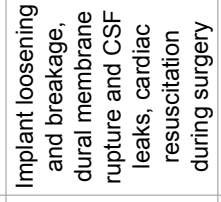 & 1 & 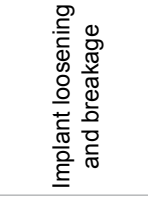 & 1 & 1 & 1 & 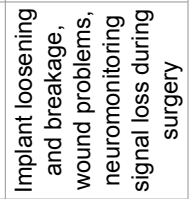 \\
\hline 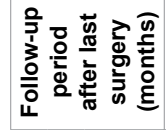 & is & $\hat{\theta}$ & f & $\stackrel{9}{\longrightarrow}$ & N & $\wedge$ & $\wedge$ & $\stackrel{\text { N }}{2}$ & N \\
\hline 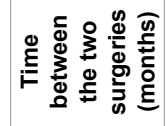 & $\wedge$ & $\stackrel{\sim}{\sim}$ & $\stackrel{N}{N}$ & 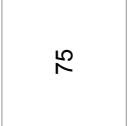 & - & 0 & $\stackrel{\text { A }}{ }$ & N & $\stackrel{\circ}{\circ}$ \\
\hline 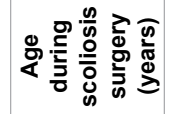 & $\stackrel{\bullet}{\stackrel{\leftrightarrow}{\sim}}$ & $\begin{array}{l}\infty \\
\stackrel{\infty}{\rho} \\
\stackrel{\rho}{n}\end{array}$ & $\stackrel{\infty}{N}$ & $\stackrel{\circ}{\stackrel{\rho}{\Gamma}}$ & $\stackrel{m}{=}$ & $\stackrel{\substack{\infty \\
\infty}}{\sim}$ & $\stackrel{\square}{\square}$ & 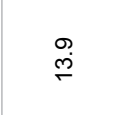 & 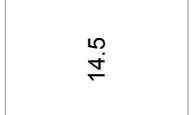 \\
\hline 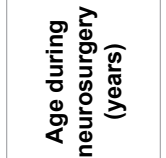 & 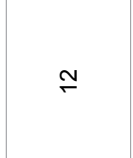 & $\stackrel{\infty}{\stackrel{\infty}{\sim}}$ & $\stackrel{\infty}{\circ}$ & $\stackrel{m}{\sim}$ & $\stackrel{\mathscr{S}}{\mathrm{N}}$ & $\stackrel{\substack{\infty \\
\infty}}{\infty}$ & 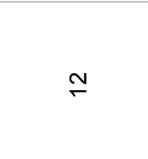 & $\stackrel{\widehat{m}}{g}$ & $\stackrel{\text { L }}{\leftarrow}$ \\
\hline 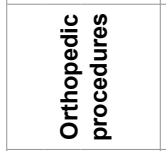 & $\begin{array}{l}\frac{u}{\omega} \\
0 \\
0\end{array}$ & $\begin{array}{c}\frac{1}{0} \\
0 \\
0\end{array}$ & $\begin{array}{l}0 \\
0 \\
0 \\
0 \\
w \\
0 \\
0\end{array}$ & $\begin{array}{l}\frac{0}{0} \\
\stackrel{0}{u} \\
0 \\
0 \\
0\end{array}$ & $\begin{array}{l}0 \\
0 \\
0 \\
\frac{1}{w} \\
0 \\
0\end{array}$ & $\begin{array}{l}\frac{u}{0} \\
0 \\
\alpha\end{array}$ & $\begin{array}{l}0 \\
0 \\
0 \\
\frac{1}{4} \\
0 \\
0\end{array}$ & $\begin{array}{l}0 \\
\frac{1}{0} \\
w \\
0 \\
0\end{array}$ & $\begin{array}{l}0 \\
0 \\
0 \\
u \\
w \\
0\end{array}$ \\
\hline 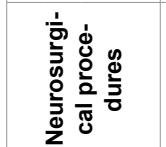 & 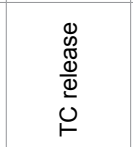 & 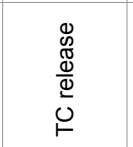 & 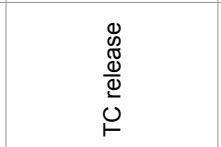 & 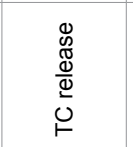 & 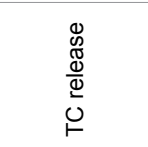 & 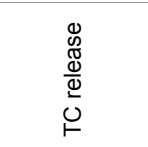 & 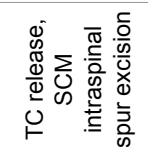 & 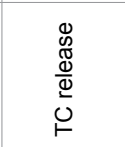 & 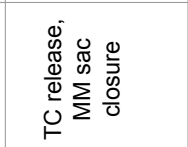 \\
\hline 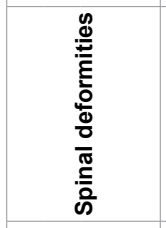 & 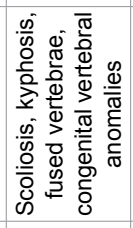 & 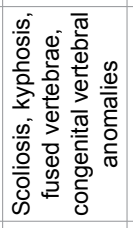 & 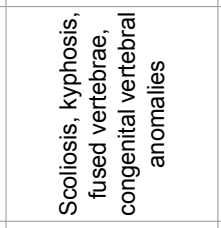 & 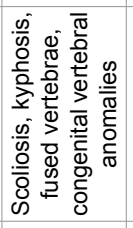 & 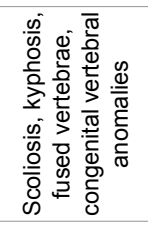 & 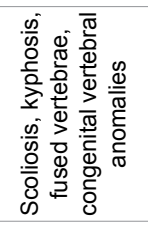 & 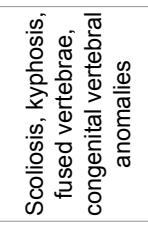 & 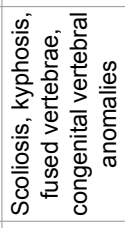 & 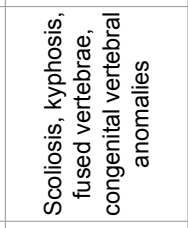 \\
\hline 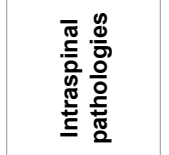 & $\stackrel{0}{\circ}$ & $\stackrel{0}{\vdash}$ & 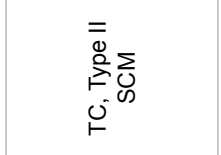 & $\stackrel{0}{\vdash}$ & 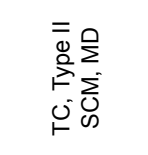 & $\stackrel{0}{\vdash}$ & 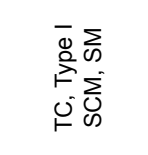 & 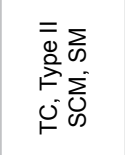 & $\begin{array}{l}\sum_{\infty} \\
\sum_{0}^{0} \\
0 \\
1\end{array}$ \\
\hline 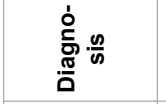 & os & 3 & 8 & is & 乏 & 8 & o & O) & $\stackrel{\rho}{\Sigma}$ \\
\hline ๑̊㐅. & L & ч & \llcorner & \llcorner & $\Sigma$ & ч & \llcorner & $\Sigma$ & \llcorner \\
\hline$\stackrel{0}{z}$ & - & $N$ & $m$ & $\checkmark$ & $\infty$ & 0 & $r$ & $\infty$ & 0 \\
\hline
\end{tabular}




\begin{tabular}{|c|c|c|c|c|c|c|c|c|c|}
\hline ฯ & ơ & $\hat{\Upsilon}$ & 号 & $\uparrow$ & $\bar{y}$ & $\bar{y}$ & q & $\bar{\zeta}$ & Fे \\
\hline$\stackrel{2}{\circ}$ & $\bar{m}$ & $\stackrel{\mathscr{N}}{\sim}$ & is & $\stackrel{\circ}{1}$ & ळ & J & \& & 8 & g \\
\hline 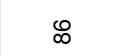 & $\varnothing_{1}$ & $\bar{\infty}$ & 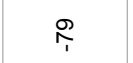 & $\bar{\infty}$ & 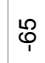 & 象 & ț & $\tilde{\varphi}$ & $\propto_{1}$ \\
\hline$\varphi$ & ㄱ & ঙิ & $\cong$ & 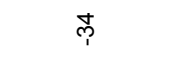 & $\hat{\emptyset}$ & $\widetilde{0}$ & 呙 & \& & g \\
\hline$\stackrel{9}{\leftarrow}$ & $\mathfrak{N}$ & $F$ & $\stackrel{\infty}{+}$ & $\stackrel{\infty}{+}$ & $\hat{N}$ & 웅 & \& & o & $\stackrel{\nabla}{\square}$ \\
\hline$\stackrel{\llcorner}{N}$ & $\stackrel{\omega}{\stackrel{0}{\circ}}$ & $\approx$ & ঃ & $\stackrel{8}{\circ}$ & 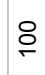 & $\mathcal{F}$ & $\bar{r}$ & \& & $R$ \\
\hline i & 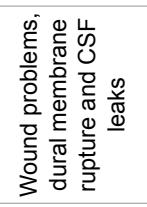 & i & : & 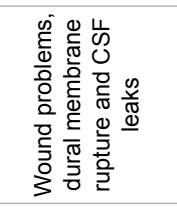 & i & i & i & 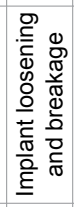 & : \\
\hline 0 & $\bar{\infty}$ & $\approx$ & $\stackrel{\square}{\square}$ & $\stackrel{\circ}{\circ}$ & $\stackrel{\infty}{\sim}$ & q & $\stackrel{\infty}{\infty}$ & $\widetilde{N}$ & $\approx$ \\
\hline$\frac{0}{\frac{9}{J}}$ & $\stackrel{\infty}{\sim}$ & $\Sigma$ & $\stackrel{0}{+}$ & $\stackrel{\widehat{N}}{N}$ & A & 0 & 0 & $\stackrel{\infty}{\infty}$ & 0 \\
\hline$\cong$ & $\stackrel{9}{\leftarrow}$ & 0 & $\stackrel{\text { }}{\stackrel{\sim}{r}}$ & $\hat{o}$ & $\hat{\check{F}}$ & $\begin{array}{l}\stackrel{\leftrightarrow}{\oplus} \\
\stackrel{\oplus}{\circ}\end{array}$ & 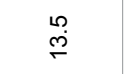 & 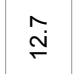 & $\underset{\mathscr{O}}{\mathscr{O}}$ \\
\hline$\stackrel{m}{\circ}$ & $\bar{o}$ & $\overline{0}$ & '̇ & $\overline{0}$ & مُ & $\begin{array}{l}0 \\
\dot{0} \\
\dot{\varphi}\end{array}$ & $\stackrel{m}{c}$ & 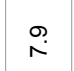 & $\begin{array}{c}N \\
\mathscr{\sigma}\end{array}$ \\
\hline $\begin{array}{l}\frac{1}{0} \\
0 \\
\alpha\end{array}$ & $\begin{array}{l}\text { 峈 } \\
0\end{array}$ & 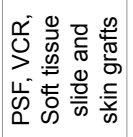 & 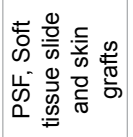 & $\begin{array}{l}0 \\
0 \\
0 \\
4 \\
w \\
0 \\
0\end{array}$ & $\begin{array}{l}\omega \\
\omega \\
0\end{array}$ & $\begin{array}{l}u \\
\omega \\
0 \\
\alpha\end{array}$ & $\begin{array}{l}\text { un } \\
0 \\
0 \\
\overline{0} \\
\underline{0} \\
0\end{array}$ & $\begin{array}{l}\bar{\Phi} \\
\mathbb{U} \\
\mathbb{0}\end{array}$ & $\begin{array}{l}0 \\
0 \\
0 \\
u \\
w \\
0 \\
0\end{array}$ \\
\hline 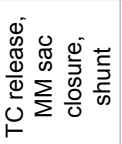 & 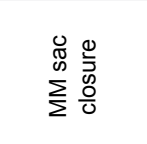 & 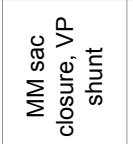 & 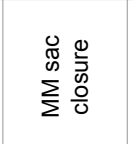 & 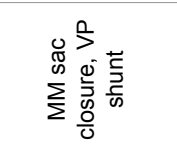 & 믐 & 这 & 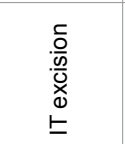 & 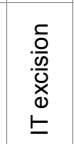 & 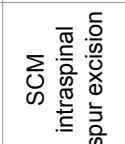 \\
\hline 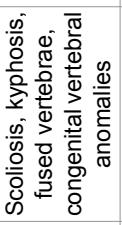 & 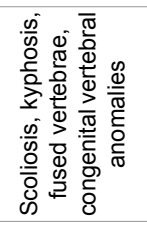 & 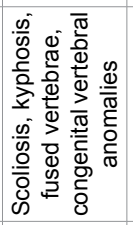 & 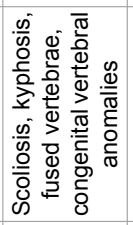 & 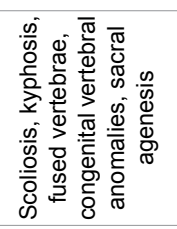 & $\begin{array}{l}\frac{0}{0} \\
0 \\
\frac{0}{2} \\
\frac{1}{1} \\
\frac{0}{0} \\
\frac{0}{0} \\
i \\
i\end{array}$ & $\begin{array}{l}\frac{0}{0} \\
\frac{0}{0} \\
\frac{0}{2} \\
\frac{1}{3} \\
\frac{0}{0} \\
\frac{0}{0} \\
\frac{0}{0} \\
i\end{array}$ & 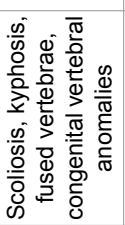 & 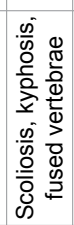 & 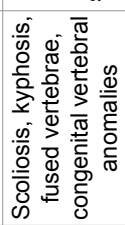 \\
\hline 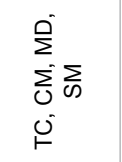 & 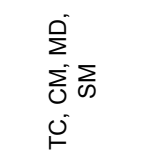 & $\begin{array}{l}\sum_{0}^{0} \\
\sum_{0}^{\infty} \sum_{\infty} \\
0 \\
1\end{array}$ & $\begin{array}{l}\sum_{0}^{0} \\
\sum_{0}^{\infty} \sum_{\infty}^{\infty} \\
0 \\
1\end{array}$ & 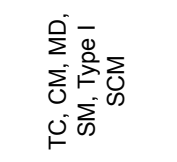 & $\begin{array}{l}\sum_{0} \\
\sum \\
\text { j }\end{array}$ & $\begin{array}{l}\sum_{0} \\
\sum_{0}\end{array}$ & 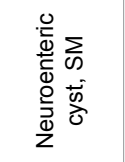 & 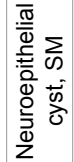 & 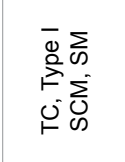 \\
\hline$\stackrel{O}{\Sigma}$ & $\stackrel{O}{\Sigma}$ & $\stackrel{Q}{\Sigma}$ & $\stackrel{Q}{\Sigma}$ & $\stackrel{\rho}{\Sigma}$ & $\underline{\omega}$ & $\underline{\Omega}$ & $\bullet$ & เ & $\ddot{0}$ \\
\hline ч & ᄂ & $\Sigma$ & $\Sigma$ & ц & $\Sigma$ & $\Sigma$ & ч & ц & ч \\
\hline$\stackrel{0}{\circ}$ & $=$ & $\simeq$ & $\stackrel{m}{=}$ & \pm & 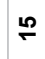 & $\stackrel{0}{\circ}$ & 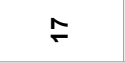 & $\stackrel{\infty}{=}$ & $\stackrel{9}{9}$ \\
\hline
\end{tabular}



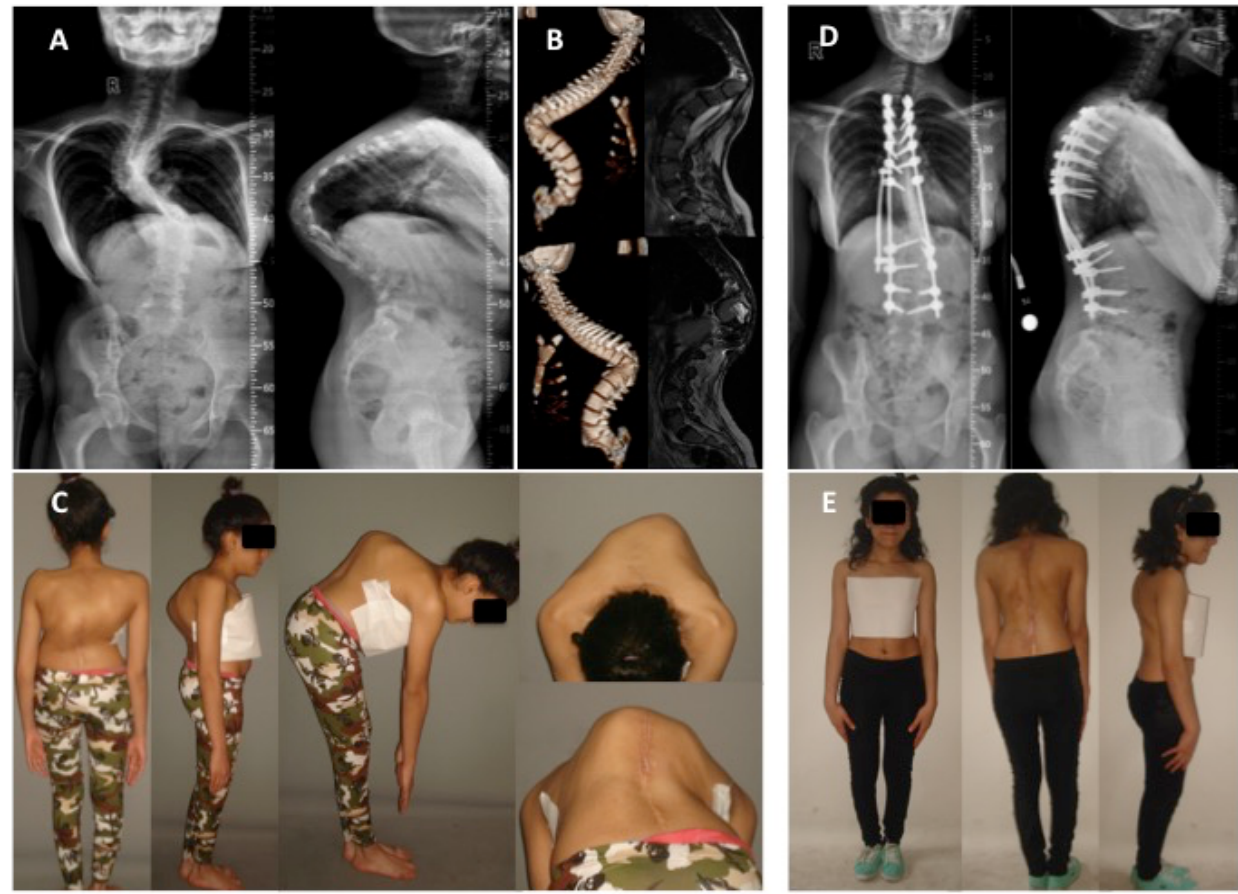

Figure 1: A female patient (17.5-year-old) underwent surgery due to intraspinal anomalies caused by congenital kyphoscoliosis and myelodysplasia. The myelomeningocele sac was closed. Associated anomalies on MRI were tethered cord and hydromyelia. Neurosurgical treatment was performed for tethered cord ( 14.5 years); posterior spinal fusion and pedicle subtraction osteotomy were performed seven months later. The complication of intraoperative development of the loss of neuromonitorization was corrected. Postoperative revision took place seven months later due to rod fracture development. The follow-up period after the last surgery was 22 months. (A-C) Preoperative X-ray, CT, MRI and clinical images. (D and E) Views from the final radiological and clinical examinations

lacerations occurred in two cases during surgery and were repaired. In a case with congenital kyphoscoliosis deformity, the dural damage which developed after PSO and cerebrospinal fluid leakage (CFL) was repaired. Soft tissue infection that developed in two cases was treated with early-period wound debridement. Postoperative bracing was applied on the patients for six months.

Two patients (female) had scoliosis deformities associated with intraspinal tumors. Posterior instrumentation with deformity correction was applied in both cases after tumor excision during separate sessions. Intradural neurenteric cysts (syringomyelia and congenital vertebra anomalies) and neuroepithelial cysts (syringomyelia) were accompanied by scoliosis and kyphosis deformities in these patients. Mean age of the patients at the time of neurosurgery was 10.5 (range: 7.9 to 13) years. The period between the two surgeries was measured a mean of 32 (range: 6 to 58) months and the follow-up period from the deformity surgery was measured 4.1 (range: 2.6 to 5.5 ) years on average. The preoperative AP Cobb angle of $67.5^{\circ}$ (range: $64^{\circ}$ to $71^{\circ}$ ) was measured $37.5^{\circ}$ (range: $30^{\circ}$ to $45^{\circ}$ ) at the final follow-up with $45 \%$ (range: $46.8 \%$ to $63.3 \%$ ) correction. On the sagittal plane, the preoperative TK was $54.5^{\circ}$ (range: $53^{\circ}$ to $-56^{\circ}$ ). This value improved to $54^{\circ}$ (range: $45^{\circ}$ to $-63^{\circ}$ ) at the final follow-up. A second operation was performed on one patient for proximal junction kyphosis, which developed due to implant insufficiency. No problems were observed on either of these patients at the final examination.

In Type I SCM patients ( 2 patients), deformity correction was applied with intraspinal spur excision by neurosurgery. In one case with Type I SCM (diastematomyelia) and scoliosis deformity (16.2-yearold female), posterior deformity surgery and PSO (T12 level) were performed concurrently for intraspinal spur excision and scoliosis deformity. In another case with Type I SCM accompanied by tethered cord and syringomyelia (twelve-year-old female), tethered cord release and intraspinal spur excision was performed, followed by posterior deformity surgery and PSO 16 months later. Mean age of the patients during neurosurgery was 14.1 (range: 12 to 16.2 ) years. The mean period between the two surgeries was calculated as 12 (range: 0 to 24) months and the mean follow-up period as 1.9 (range: 1.3 to 2.5 ) years. The preoperative AP Cobb angle of $73.5^{\circ}$ (range: $70^{\circ}$ to $77^{\circ}$ ) was measured $17.5^{\circ}$ (range: $14^{\circ}$ to $21^{\circ}$ ) at the final examination with $76.2 \%$ correction. No postoperative complications were observed.

\section{Discussion}

During MRI studies, the incidences of intraspinal anomalies (CM, syringomyelia, diastematomyelia and tethered cord) have been noted as 15 to $43 \%$ in congenital scoliosis $[1,18]$ and 20 to $38 \%[2,3]$ in infantile and juvenile scoliosis patients. These incidences were also found to be low in adolescent idiopathic scoliosis (AIS) patients [8]. Most intraspinal anomalies (such as small syrinx or minimal cerebellar ectopia) may not require surgical treatment [19]. Even though reported abnormal MRI findings in AIS patients which require no neurosurgical intervention are between 2.1 and 15\%, the incidence of intraspinal anomaly that requires surgery in infantile scoliosis has been reported as high as $50 \%[20]$ in the literature.

\section{Chiari malformation and syringomyelia}

Chiari malformation observed on MRI has been described as a cerebellar tonsillar herniation of $>4-5 \mathrm{~mm}$ from the foramen magnum downwards [11]. Scoliosis has been recorded to be present in $80 \%$ of CM-I patients [5-9]. It has been shown that decompression at an early age, when the degree of the curve is small, is greatly beneficial to the correction of a scoliosis curve whereas intervention at an older age and at a later time is not effective [17,21-24].

In our study, there was no improvement in scoliosis after the PFD surgery. This was thought to be due to the late neurosurgical 
intervention, as stated in literature. Therefore, there was no difference between the results of the two patients who underwent surgery for CM-1 malformation and scoliosis/kyphosis deformity, either with PFD and posterior fusion/deformity correction during the same session or in two stages. If PFD surgery is not done until adolescence, simultaneous PFD decompression and posterior fusion/deformity correction surgery can be done.

\section{Tethered cord}

The risk of neurological injury may increase during scoliosis surgery, with tethered cord incidence as high as $20 \%$ in infantile, juvenile and congenital forms. Stopping or correcting the associated scoliosis in the early period may be possible with tethered cord treatment [13]. The main factors which cause curve progression are a curvature of $>40^{\circ}$, skeletal immaturity, thoracic curve, and curves which cause lower lumbar and sacral motor dysfunction.

Spinal deformities may be corrected in around 6 weeks to 6 months after the release. Performance of the surgery 4 to 6 weeks before scoliosis correction has been shown to reduce the potential of recurrent tethering during the recovery period [19]. However, there is no specific data on the timing of surgery $[14,19]$. With the popularization of intraoperative neuromonitorization, the performance of both procedures during with a single application of anesthesia has become possible [3,15,19,25-28].

In our study, tethered cord release was required in 10 of the 15 patients with tethered cord. Eight of these patients were treated with two-stage and two with concurrent release. A shorter duration of stay in hospital, lower cost and faster recovery were achieved with single-stage treatment when compared with the two-stage surgery. However, the use of this concurrent approach should be meticulously considered for the selection of patients who could benefit from it [15].

\section{Myelodysplasia}

Chiari malformation and tethered cord pathologies, scoliosis and kyphosis deformity may be observed together with myelomeningocele. Release of the spinal cord tension is recommended before deformity correction due to the large-scale and progressive curves $[13,19]$. Significant clinical correction after tethered cord release was in the lower extremities rather than sphincters, as reported in the literature [29].

In our study, postnatally-open myelomeningocele sacs were closed with neurosurgical interventions in six of the seven cases suffering from myelodysplasia. Due to the poor sitting balance and progressive deformities on the coronal/sagittal planes, the patients/cases had to be treated surgically. During the postoperative early and final controls, an obvious correction was obtained in spinal deformities and in sitting balance; however, a significant change was not observed in the motor functions of the lower extremities.

\section{Split Cord Malformation (SCM)}

Split cord malformation is an anomaly related with embryological etiologies that can be defined as double spinal cords. The symptoms of SCM are spinal dysraphism, scoliosis or kyphoscoliosis, bowel and bladder incontinence, foot deformities, spasticity, weakness, paresthesia, and walking anomalies [13]. With SCM, scoliosis is observed in approximately $40 \%$ of the patients and to a greater degree in older children. [30] Meanwhile, progressive neurological disorder and agerelated risk increases were shown in 50\% of the patients [31]. Therefore, all SCM patients must be carefully assessed before the development of orthopedic/neurological deterioration or spinal instrumentation, and hemicords must surgically be released [31-33].

In Type I SCM patients (two patients), the deformity correction was performed concurrently or separately with intraspinal spur excision by neurosurgery. Patients who did not face any extra complications had a shorter period of hospitalization and faster recovery.

Sixteen of our cases were treated with neuromonitorization whereas the neurological conditions of the first three cases where checked using the wake-up test without neuromonitorization. In one patient, after neuromonitorization signal loss, the release of the rods re-normalized the signals and the correction was completed without any extra negative circumstances. No early or late neurological negative circumstances were observed in other patients. A more reliable correction and posterior fusion was obtained with concurrent surgeries and neuromonitorization. Successive surgical intervention for intraspinal pathologies and deformity correction in one session may provide a shorter duration of stay in hospital and recovery period compared to two-stage surgery.

\section{Conclusion}

The main limitations of our study were the small number of patients and lack of a standard approach for surgical treatments applicable to intraspinal anomalies and spinal deformities. In high-angle spinal deformities, repeated releases may be required due to tethered cord cohesiveness, particularly before the correction of the deformity. Making a decision according to factors such as etiology, deformity size, curve progression, and the patient's age is recommended when planning the two-stage surgery of serious spinal curves associated with intraspinal pathologies. Concurrent surgical interventions for both problems may be preferable for eligible patients due to shorter duration of stay in hospital and quicker recovery with no complications.

\section{References}

1. Belmont PJ, Kuklo TR, Taylor KF, Freedman BA, Prahinski JR, et al. (2004) Intraspinal anomalies associated with isolated congenital hemivertebra: The role of routine magnetic resonance imaging. J Bone Joint Surg Am 86: 1704-1710.

2. Dobbs MB, Lenke LG, Szymanski DA, Morcuende JA, Weinstein SL, et al. (2002) Prevalence of neural axis abnormalities in patients with infantile idiopathic scoliosis. J Bone Joint Surg Am 84: 2230-2234.

3. Hamzaoğlu A, Öztürk C, Tezer M, Aydoğan M, Sarıer M, et al. (2007) Simultaneous surgical treatment in congenital scoliosis and/or kyphosis associated with intraspinal abnormalities. Spine (Phila Pa 1976) 32: 2880-2884

4. Davids JR, Chamberlin E, Blackhurst DW (2004) Indications for magnetic resonance imaging in presumed adolescent idiopathic scoliosis. J Bone Joint Surg Am 86: 2187-2195.

5. Eule JM, Erickson MA, O'Brien MF, Handler M (2002) Chiari I malformation associated with syringomyelia and scoliosis: A twenty-year review of surgical and nonsurgical treatment in a pediatric population. Spine (Phila Pa 1976) 27: $1451-1455$.

6. Hankinson TC, Klimo P Jr, Feldstein NA, Anderson RC, Brockmeyer D (2007) Chiari malformations, syringohydromyelia and scoliosis. Neurosurg Clin N Am 18: $549-568$.

7. Ono A, Ueyama K, Okada A, Echigoya N, Yokoyama T, et al. (2002) Adult scoliosis in syringomyelia associated with Chiari I malformation. Spine (Phila Pa 1976) 27: E23-E28.

8. Krieger MD, Falkinstein Y, Bowen IE, Tolo VT, McComb JG (2011) Scoliosis and chiari malformation type I in children: Clinical article. J Neurosurg Pediatr $7: 25-29$

9. Strahle J, Muraszko KM, Kapurch J, Bapuraj JR, Garton HJ, et al. (2011) Chiari malformation type I and syrinx in children undergoing magnetic resonance imaging. J Neurosurg Pediatr 8: 205-213.

10. Charry O, Koop S, Winter R, Lonstein J, Denis F, et al. (1994) Syringomyelia 
Citation: Balioglu MB, Kargin D, Albayrak A, Atici Y, Oner A, et al. (2018) Surgical Treatment Approaches in Severe Spinal Deformities Associated with Intraspinal Pathologies. J Spine 7: 417. doi: 10.4172/2165-7939.1000417

and scoliosis: A review of twenty-five pediatric patients. J Pediatr Othop 14: 309-317.

11. Farley FA, Song KM, Birch JG, Browne R (1995) Syringomyelia and scoliosis in children. J Pediatr Orthop 15: 187-192.

12. Mooney JF, Glazier SS, Barfield WR (2012) Concurrent orthopedic and neurosurgical procedures in pediatric patients with spinal deformity. J Pediatr Orthop B 21: 602-605.

13. Cardoso M, Keating RF (2009) Neurosurgical management of spinal dysraphism and neurogenic scoliosis. Spine (Phila Pa 1976) 34: 1775-1782.

14. Ayvaz M, Alanay A, Yazıcı M, Acaroglu E, Akalan N, et al. (2007) Safety and efficacy of posterior instrumentation for patients with congenital scoliosis and spinal dysraphism. J Pediatr Orthop 27: 380-386.

15. Mehta VA, Gottfried ON, McGirt MJ, Gokaslan ZL, Ahn ES, et al. (2011) Safety and efficacy of concurrent pediatric spinal cord untethering and deformity correction. J Spinal Disord Tech 24: 401-405.

16. Bhangoo R, Sgouros S (2006) Scoliosis in children with chiari I-related syringomyelia. Childs Nerv Syst 22: 1154-1157

17. Ozerdemoglu RA, Denis F, Transfeldt EE (2003) Scoliosis associated with syringomyelia: Clinical and radiologic correlation. Spine (Phila Pa 1976) 28 1410-1417.

18. McMaster MJ (1984) Occult intraspinal anomalies and congenital scoliosis. J Bone Joint Surg Am 66: 588-601.

19. Samdani AF, Asghar J, Pahys J, D'Andrea L, Randal R (2007) Betz RRConcurrent spinal cord untethering and scoliosis correction: Case report. Spine (Phila Pa 1976) 32: E832-E836

20. Gupta P, Lenke LG, Bridwell KH (1998) Incidence of neural axis abnormalities in infantile and juvenile patients with spinal deformity. Is a magnetic resonance image screening necessary? Spine (Phila Pa 1976) 23: 206-210.

21. Flynn JM, Sodha S, Lou JE, Adams SB Jr, Whitfield B, et al. (2004) Predictors of progression of scoliosis after decompression of an Arnold Chiari I malformation. Spine (Phila Pa 1976) 29: 286-292.

22. Ozerdemoglu RA, Transfeldt EE, Denis F (2003) Value of treating primary causes of syrinx in scoliosis associated with syringomyelia. Spine (Phila Pa 1976) 28: 806-814
23. Sengupta DK, Dorgan J, Findlay GF (2000) Can hindbrain decompression for syringomyelia lead to regression of scoliosis? Eur Spine J 9: 198-201.

24. Brockmeyer D, Gollogly S, Smith JT (2003) Scoliosis associated with Chiar I malformations: The effect of suboccipital decompression on scoliosis curve progression: A preliminary study. Spine (Phila Pa 1976) 28: 2505-2509.

25. Luk KD, Hu Y, Wong YW, Cheung KMC (2001) Evaluation of various evoked potential techniques for spinal cord monitoring during scoliosis surgery. Spine (Phila Pa 1976) 26: 1772-1777.

26. MacDonald DB, Al Zayed Z, Khoudeir I, Stigsby B (2003) Monitoring scoliosis surgery with combined multiple pulse transcranial electric motor and cortical somatosensory-evoked potentials from the lower and upper extremities. Spine (Phila Pa 1976) 28: 194-203.

27. Paradiso G, Lee GY, Sarjeant R, Hoang L, Massicotte EM, et al. (2006) Multimodality intraoperative neurophysiologic monitoring findings during surgery for adult tethered cord syndrome: Analysis of a series of 44 patients with long-term follow-up. Spine (Phila Pa 1976) 31: 2095-2102.

28. Bowman RM, Mohan A, Ito J, Seibly JB, McLone DG (2009) Tethered cord release: A longterm study of 114 patients. J Neurosurg Pediatr 3: 181-187.

29. Daszkiewicz P, Barszcz S, Roszkowski M, Maryniak A (2007) Tethered cord syndrome in children-impact of surgical treatment on functional neurological and urological outcome. Neurol Neurochir Pol 41: 427-435.

30. Hilal SK, Marton D, Pollack E (1974) Diastematomyelia in children: Radiographic study of 34 cases. Radiology 112: 609-621.

31. Pang D, Dias MS, Ahab-Barmada M (1992) Split cord malformation. Part I: A unified theory of embryogenesis for double spinal cord malformations. Neurosurgery 31:451-480.

32. Ersahin Y, Mutluer S, Kocaman S, Demirtaş E (1998) Split spinal cord malformations in children. J Neurosurg 88: 57-65.

33. Keating RF, Goodrich JT (1998) Surgical management of tethered cord: Diastamatomyelia, hypertrophied filum terminale, myelomeningocele. In: Rengachary SS, Willkins R, editors. Neurosurgical operative atlas. American Association of Neurological Surgeons Publications, pp: 211-218. 